\title{
Spatial Distribution of Flexural Strength in Y-Ba-Cu-O Bulk Superconductors
}

\author{
K. Y. Huang, D. Zhou, J. Srpčič, Y. Shi, D. K. Namburi, A. Dennis, M. D. Ainslie, Senior Member, IEEE, M. Boll,
} R. Bause, M. Filipenko, J. H. Durrell and D. A. Cardwell

\begin{abstract}
In order to determine the spatial distribution of the flexural strength, three-point bend tests have been performed on bar specimens cut from four $\mathrm{YBa}_{2} \mathrm{Cu}_{3} \mathrm{O}_{7-\delta}$ single-grain bulk superconductors. A relatively large spread of the room temperature flexural strength, average and standard deviation of 49.3 MPa and 12.7 MPa respectively, was observed across the four bulk samples. This is attributed to the systemic microstructural variation introduced by the seeded melt growth. In particular, the strength was shown to be related to the local porosity and $\mathrm{Y}_{2} \mathrm{BaCuO}_{5}$ content. To further examine bulk-tobulk variability, indirect tensile (Brazilian) tests were conducted on twelve $16 \mathrm{~mm}$ diameter bulk superconductors from three production batches. The Weibull modulus for these was calculated to be 8.76, suggesting that, despite the large spread in strength with position within a bulk, the batch processing of bulk superconductors can consistently yield samples with comparable overall mechanical strengths.
\end{abstract}

Index Terms - Yttrium barium copper oxide, superconducting magnets, flexural strength, Weibull distribution

\section{INTRODUCTION}

$\mathrm{T}$ $\mathrm{HE}(\mathrm{RE}) \mathrm{Ba}_{2} \mathrm{Cu}_{3} \mathrm{O}_{7-\delta}$ family [where $\mathrm{RE}$ denotes rare earth elements, such as Y, Gd and $\mathrm{Sm}$ ] of melt processed bulk high temperature superconductors has outstanding technological potential, most notably for application as quasipermanent high-trapped-field magnets[1]. This is illustrated by the record trapped field of $17.6 \mathrm{~T}$ at $26 \mathrm{~K}$ between a stack of two $25 \mathrm{~mm}$ diameter $\mathrm{GdBa}_{2} \mathrm{Cu}_{3} \mathrm{O}_{7-\delta}$ bulk superconductors[2]. However, significant magnetic stresses due to the large Lorentz force are generated during the magnetization and operation of such trapped-field magnets. This leads to cracking and failure, therefore making their mechanical properties, rather than the critical current or grain size, the critical, performance-limiting factor at high fields of above around $8 \mathrm{~T}[3-6]$.

Practical applications of bulk superconductors are further complicated by the nature of the melt growth process[7],

Automatically generated dates of receipt and acceptance will be placed here; authors do not produce these dates. This work was supported by Siemens AG, Corporate Technology, Munich, Germany. The work of Y. Shi and A. Dennis were supported by an Engineering and Physical Sciences Research Council Grant EP/P00962X/1. The work of M. D. Ainslie was supported in part by a Royal Academy of Engineering Research Fellowship and in part by an Engineering and Physical Sciences Research Council (EPSRC) Early Career Fellowship EP/P020313/1. All data are provided in full in the results section of this paper.

K. Y. Huang, D. Zhou, J. Srpčič, Y. Shi, D. K. Namburi, A. Dennis, M. D. Ainslie, J. H. Durrell and D. A. Cardwell are with the Bulk Superconductivity Group, Department of Engineering, University of Cambridge, Cambridge, UK (e-mail: kyh30@cam.ac.uk).

M. Boll, R. Bause and M. Filipenko are with Siemens AG, Corporate Technology, Munich, Germany (e-mail: martin.boll@ siemens.com) which produces an inhomogeneous microstructure within a bulk single grain. For instance, systemic variations in the local porosity and the local $\mathrm{RE}_{2} \mathrm{BaCuO}_{5}$ (RE-211) content with distance from the seed have been observed[8-13]. In addition, the volume fraction of RE-211 in the $a$-growth direction tends to be higher than that of the $c$-growth direction due to the difference in the critical radius for each direction [9, 14]. Previous studies have shown a dependence of the mechanical properties on regional porosity as well as the initial RE-211 fraction of the precursor powders[15-22].

In the present study, the spatial distribution of the flexural strength in four $\mathrm{YBa}_{2} \mathrm{Cu}_{3} \mathrm{O}_{7-\delta} \quad(\mathrm{YBCO})$ single-grains approximately $25 \mathrm{~mm}$ in diameter, two fabricated via top seeded melt growth (TSMG)[23] and two via top seeded infiltration and growth (TSIG)[24], was investigated via the three-point bend test and related to the underlying microstructure.

Additionally, the indirect tensile test, also known as the Brazilian test[25], was carried out on three batches of YBCO bulks $16 \mathrm{~mm}$ in diameter to determine the sample-to-sample variation in the strength of bulks as a whole. The results from two different test techniques would allow for a comparison of the strength distribution within a single bulk to the bulk-tobulk scatter.

\section{EXPERIMENTAL METHODS}

Two sizes of single-grain bulk superconductors were fabricated (diameter of $25 \mathrm{~mm}, 16 \mathrm{~mm}$ and thickness of $10 \mathrm{~mm}$, $6 \mathrm{~mm}$ respectively) in air via top seeded melt growth from $75 \%$ $\mathrm{YBa}_{2} \mathrm{Cu}_{3} \mathrm{O}_{7}+25 \% \mathrm{Y}_{2} \mathrm{BaCuO}_{5}+0.5 \% \mathrm{CeO}_{2}$ (by weight) precursors. Two $25 \mathrm{~mm}$ diameter YBCO were also fabricated via top seeded infiltration and growth[26] for the bend test. The as-grown bulk superconductors were annealed in an oxygen atmosphere to facilitate the tetragonal to orthorhombic phase transition[27]. The peak trapped fields of all samples were measured at $77 \mathrm{~K}$ with an axial Hall probe after fieldcooled magnetization with an external field of $1.2 \mathrm{~T}$, followed by mapping of the surface field to confirm single-grain structure.

\section{A. Three-Point Bend Test}

Bar specimens of dimensions $2 \mathrm{~mm} \times 1.5 \mathrm{~mm} \times 20 \mathrm{~mm}$ were cut from the $25 \mathrm{~mm}$ diameter YBCO bulks using a diamond wheel. The positions of the bars in the parent bulk and their identifying labels are shown in Fig. 1. Axial symmetry has been assumed so only half of each bulk was cut.

The samples were subsequently polished to eliminate any surface defects potentially introduced during the cutting process. The room temperature flexural strength was obtained 


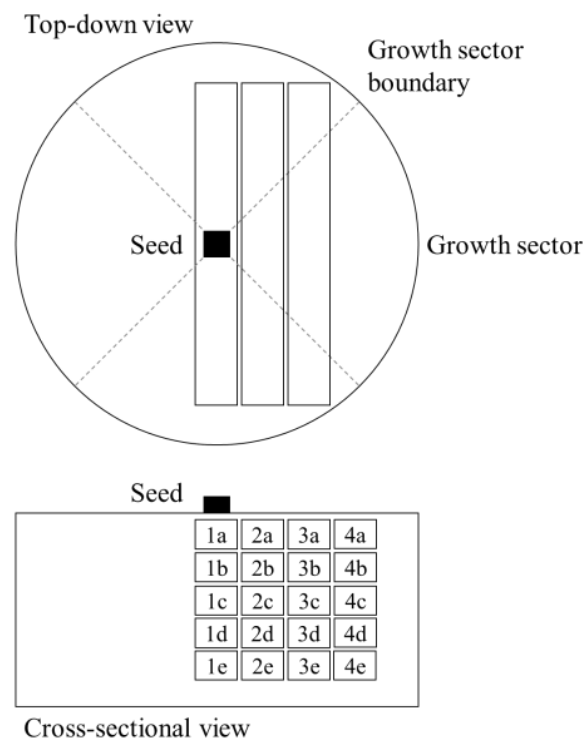

Fig. 1. Schematic illustrations of bar specimens cut from a YBCO bulk superconductor for the three-point bend test.

by applying a vertical load to the bar specimens along the crystallographic $c$-direction in an electro mechanical tensile testing machine at a crosshead speed of $0.12 \mathrm{~mm} / \mathrm{min}$, as shown in Fig. 2(a), such that the strength along the $a b$ axes could be determined. The upper $a b$ surface of each flexure bar is always subjected to tension. The flexural strength $\sigma_{f}$ was calculated using (1), where $P$ is the load at fracture, and $L, w$ and $t$ are the span length, bar width and bar thickness, respectively.

$$
\sigma_{f}=\frac{3 P L}{2 w t^{2}}
$$

The specimens were polished along the $a c$ planes and imaged under an optical microscope (with a Moticam Pro 282A CCD camera). From optical micrographs of the $a c$ planes taken at positions that correspond to the mid-point of each flexure bar (where the tensile stress would be maximum), the local porosity and Y-211 content were approximated through ImageJ image processing software[28], as an area fraction. The maximum possible error on these approximations was determined to be $\pm 10 \%$. The strength, porosity and Y-211 content for each position are shown in Table I.

\section{B. Indirect Tensile Test}

Twelve $16 \mathrm{~mm}$ diameter $6 \mathrm{~mm}$ thick YBCO bulks were (a)

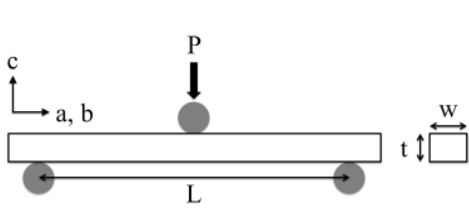

(b)

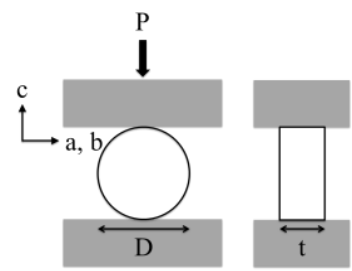

Fig. 2. Schematic illustrations of the (a) three-point bend test on bar specimens and (b) indirect tensile test on cylindrical specimens. The crystallographic directions are shown.

grown in three batches from the same precursor powders and with the same heating profile. The circumferential surfaces of the cylindrical bulks were subsequently polished to eliminate irregularities. They were compressed along growth sectors under flat platens in a tensile testing machine, as shown in Fig. 2(b), at a rate of $0.01 \mathrm{~mm} / \mathrm{min}$ to avoid exceeding a stress rate of $0.35 \mathrm{MPa} / \mathrm{s}$, until a hairline crack formed along the principal axis due to induced tensile stress in the perpendicular direction[25, 29]. The indirect tensile strength $\sigma_{i d s}$ was calculated using (2), where $P$ is the load at fracture, and $D$ and $t$ are the diameter and thickness, respectively.

$$
\sigma_{i d s}=\frac{2 P}{\pi D t}
$$

\section{RESULTS AND DISCUSSION}

\section{A. Spatial Distribution of the Flexural Strength}

Fig. 3 (a) and (b) show the spatial distribution of the average flexural strength measured at each position for four 25 $\mathrm{mm}$ diameter YBCO bulks, one of which was supplied by CAN SUPERCONDUCTORS. It is evident from Fig. 3 (b) that there is a general increase in $\sigma_{f}$ with distance along the $a b$ axes from the seed, i.e. $a b$-growth sectors tend to have higher $\sigma_{f}$ than the $c$-growth sector.

The average strength of all flexure specimens is $49.3 \pm 12.7$ MPa. Applying Weibull analysis on the flexural strengths yields a Weibull modulus $m$ [30] of 4.46 across 60 flexure specimens from the four bulks, where the Weibull modulus is a dimensionless parameter typically used as a measure of the scatter and predictability of mechanical strengths, i.e. mechanical reliability. "Poor" ceramics exhibit large scatter and fall roughly in between $0<m<10$, whereas "tough" ceramics show significantly less scatter and normally are classified as $m>10$. 
(a)
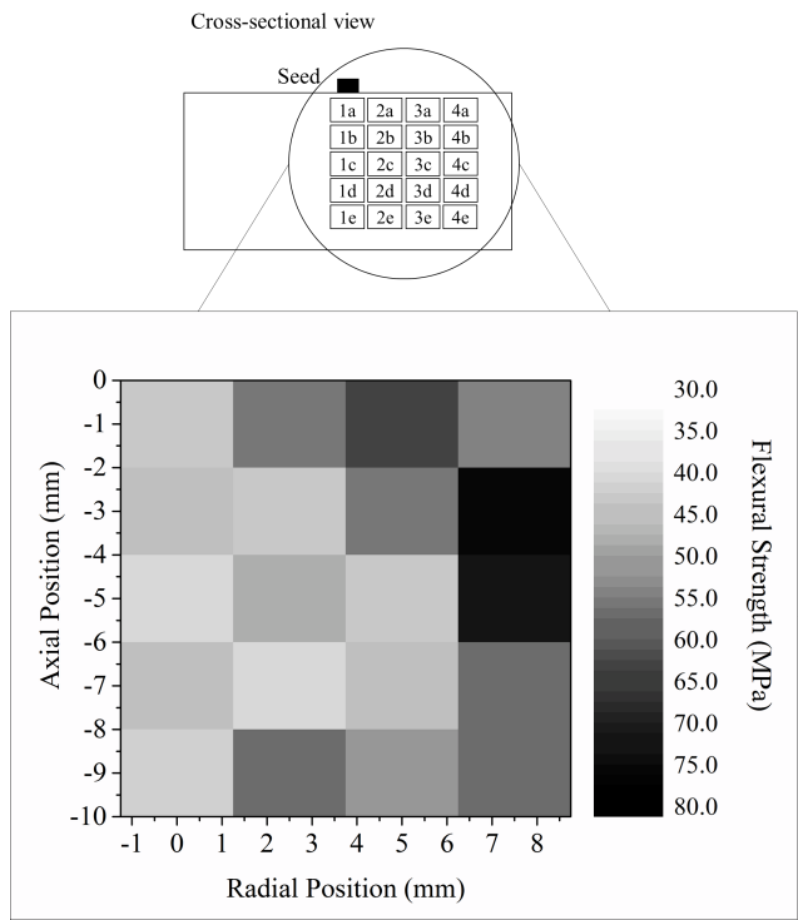

(b)

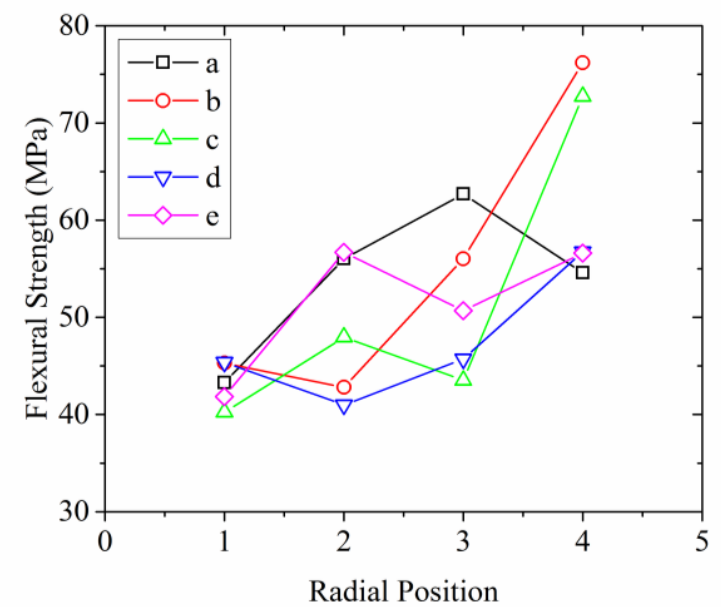

Fig. 3. (a) Cross-sectional view of the spatial distribution of room temperature flexural strength, averaged over four samples. (b) Average flexural strength as a function of position. The numerical and alphabetical labels correspond to those shown in Fig. 1.

To determine the cause of such $\sigma_{f}$ variation, $\sigma_{f}$ values were plotted against the local porosity and local Y-211 content, as shown for a bulk in Fig. 4. In order to distinguish the effects of porosity and Y-211 inclusions, the strengths were binned and averaged per $2.5 \%$ and $5 \%$ interval for the porosity and $\mathrm{Y}$ 211 content respectively. This is due to the fact at any given porosity, Y-211 content may not be a fixed variable, and vice versa.

The relationships observed are consistent with previous reports that have shown firstly, an increase in mechanical strength with increasing RE-211 content in the preform, and secondly, a decrease in strength with increasing sample porosity. The increase in $\sigma_{f}$ with Y-211 content can be explained by the fact that the high stiffness Y-211 particle (a)

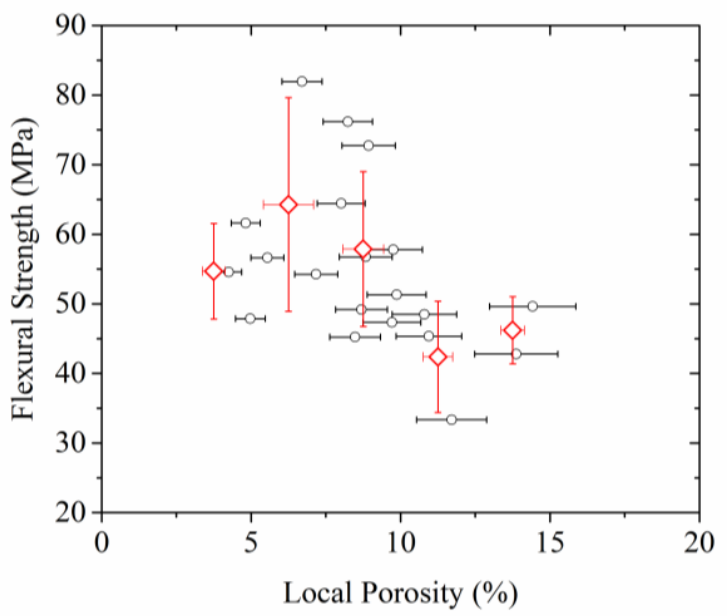

(b)

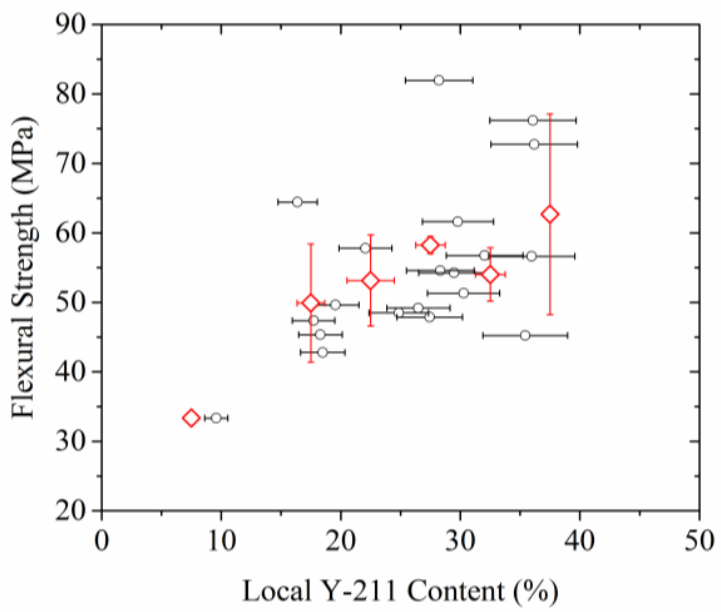

Fig. 4. Flexural strength as a function of local porosity and local Y-211 content for 20 flexure specimens from a YBCO bulk (black). The average strengths per $2.5 \%$ porosity and $5.0 \% \mathrm{Y}-211$ content interval are also shown (red).

inclusions tend to improve the fracture resistance, and thus toughness, of the matrix through interfacial delamination and crack bridging[15, 17, 31]. Similar reinforcement can also be provided by Ag particles[32, 33].

The decrease in $\sigma_{f}$ with porosity can be explained by the stress concentration introduced by voids within the material, causing localized regions around the voids to reach the critical stress before the nominal stress.

It has been well reported the melt growth process introduces a microstructural variation across the bulk. For instance, Y211 content tends to increase with distance along the $a b$ - and $c$-axis from the seed, as described by the particle push-trap theory[9-11], and evident from the data in Table I. It can also be seen from Table I that porosity is normally reduced at the surfaces of the bulks, since oxygen gas bubbles produced during the peritectic reaction are entrapped and coalesce into large visible pores at the center.

We believe the result is a spatial dependence of the flexural strength, which mirrors the microstructural variation, as shown in Fig. 3. This is unlike the random strength distribution normally observed in conventional sintered ceramics due to their more homogeneous microstructure. 
TABLE I

Flexural strength and the local porosity and Y-211 content at each position in a $25 \mathrm{~mm}$ diameter YBCO bulk superconductor.

\begin{tabular}{|c|c|c|c|}
\hline Position & $\begin{array}{l}\text { Flexural Strength } \\
(\mathrm{MPa})\end{array}$ & $\begin{array}{c}\text { Porosity } \\
(\%)\end{array}$ & $\begin{array}{c}\text { Y-211 Content } \\
(\%)\end{array}$ \\
\hline $1 \mathrm{a}$ & 33.3 & 11.7 & 9.6 \\
\hline $1 b$ & 64.4 & 8.0 & 16.4 \\
\hline $1 \mathrm{c}$ & 47.4 & 9.7 & 17.7 \\
\hline $1 d$ & 42.8 & 13.9 & 18.5 \\
\hline $1 \mathrm{e}$ & 45.2 & 8.5 & 35.4 \\
\hline $2 a$ & 47.9 & 5.0 & 27.4 \\
\hline $2 b$ & 48.5 & 10.8 & 24.9 \\
\hline $2 \mathrm{c}$ & 49.6 & 14.4 & 19.6 \\
\hline $2 d$ & 45.3 & 10.9 & 18.3 \\
\hline $2 \mathrm{e}$ & 51.3 & 9.9 & 30.3 \\
\hline $3 a$ & 61.6 & 4.8 & 29.8 \\
\hline $3 b$ & 82.0 & 6.7 & 28.2 \\
\hline $3 c$ & 49.2 & 8.7 & 26.5 \\
\hline $3 d$ & 57.8 & 9.8 & 22.1 \\
\hline $3 e$ & 54.3 & 7.2 & 29.5 \\
\hline $4 a$ & 54.6 & 4.3 & 28.3 \\
\hline $4 b$ & 76.2 & 8.2 & 36.1 \\
\hline $4 c$ & 72.7 & 8.9 & 36.2 \\
\hline $4 d$ & 56.7 & 8.8 & 32.0 \\
\hline $4 \mathrm{e}$ & 56.6 & 5.5 & 36.0 \\
\hline
\end{tabular}

B. Weibull Modulus from the Brazilian Test

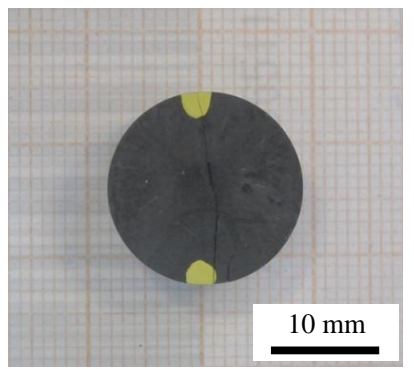

Fig. 5. Typical fracture of a $16 \mathrm{~mm}$ diameter YBCO disc from the Brazilian test. The markers indicate the direction of the applied compressive load.

The twelve $16 \mathrm{~mm}$ diameter YBCO bulks showed consistent peak trapped fields at $77 \mathrm{~K}$, with an average of $0.460 \pm 0.046 \mathrm{~T}$ at $77 \mathrm{~K}$.

Fig. 5 shows the typical failure of a Brazilian disc specimen, highlighting the expected hairline crack from the center of the cylindrical bulk due to induced tensile stress.

Fig. 6 shows the logarithmic Weibull plot of failure probability against applied tensile stress, i.e. the indirect tensile strength, at room temperature. From the slope, the Weibull modulus $m$ was determined to be 8.76 , which is comparable to $\mathrm{Al}_{2} \mathrm{O}_{3}$ engineering ceramic [34, 35]. Sample failure was assumed to follow the Weibull distribution described by (3), where $P_{f}(\sigma)$ is the cumulative failure probability at applied stress $\sigma, \sigma_{0}$ is the characteristic strength and $m$ is the Weibull modulus.

$$
P_{f}(\sigma)=1-\exp \left\lfloor-\left(\frac{\sigma}{\sigma_{0}}\right)^{m}\right\rfloor
$$

Despite high inhomogeneity within a given sample due to systemic microstructural inhomogeneity, as illustrated by $m=$ 4.46 calculated in Section III.A, the batch processing

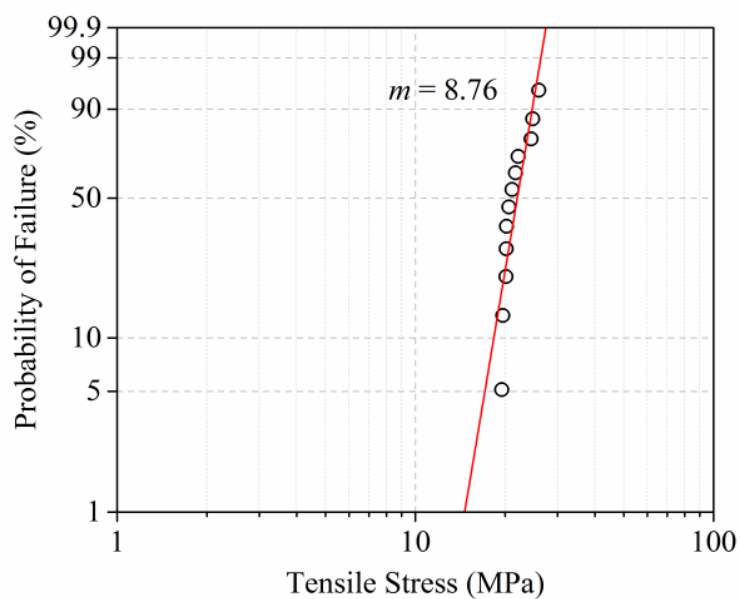

Fig. 6. Weibull plot of failure probability as a function of applied tensile stress, from twelve samples measured via the Brazilian test.

technique is shown to be capable of producing consistent defect distribution and overall mechanical strength in bulks, and therefore consistent failure probabilities, as demonstrated by a significantly higher Weibull modulus $m=8.76$.

The tensile strength measured in twelve $16 \mathrm{~mm}$ diameter YBCO bulks ranged from 19.5 $\mathrm{MPa}$ to $26.0 \mathrm{MPa}$, which agrees well with estimations from field-cooling experiments conducted by Fuchs et al., who deduced $\sigma=25 \mathrm{MPa}$ [4]. The measured tensile strengths and Weibull modulus from the Brazilian test are also more application-relevant given the magnetic stress will be distributed across the whole bulk when used as a trapped-field magnet.

\section{CONCLUSION}

The spatial distribution of flexural strength within four 25 $\mathrm{mm}$ diameter YBCO bulk superconductors was studied. From 60 flexure specimens, the average flexural strength and Weibull modulus were calculated to be $49.3 \pm 12.7 \mathrm{MPa}$ and 4.46 respectively. The strength at a given position within the bulk had correlation to the local porosity and the local Y-211 content, and this microstructural variation, introduced by the melt growth process, is in fact fairly reproducible. An understanding of this should be useful towards accurate mechanical modeling of these superconducting materials and the appropriate design of mechanical reinforcements.

In addition, the bulk-to-bulk variability was examined. The Brazilian test provides a straightforward method of determining the strength of bulk materials as-grown, i.e. as a disc. The calculated Weibull modulus of 8.76 from the Brazilian test is comparable to $\mathrm{Al}_{2} \mathrm{O}_{3}$ engineering ceramic and would imply that in spite of the large scatter of strength with position within the bulk, the mechanical failure of a bulk superconductor as a whole can be well predicted, suggesting these materials are sufficiently reliable mechanically for use as trapped-field magnets. 


\section{ACKNOWLEDGMENT}

The authors would like to thank J. Plecháček of CAN SUPERCONDUCTORS for providing a large number of samples in support of this study.

\section{REFERENCES}

1. Hull, J.R. and M. Murakami, "Applications of bulk hightemperature superconductors." Proceedings of the IEEE, 2004. 92(10): p. 1705-1718.

2. Durrell, J.H., et al., "A trapped field of $17.6 \mathrm{~T}$ in melt-processed, bulk Gd-Ba-Cu-O reinforced with shrink-fit steel." Superconductor Science and Technology, 2014. 27(8): p. 082001.

3. Ren, Y., et al., "Damage caused by magnetic pressure at high trapped field in quasi-permanent magnets composed of melttextured $\mathrm{Y}-\mathrm{Ba}-\mathrm{Cu}-\mathrm{O}$ superconductor." Physica C: Superconductivity, 1995. 251(1): p. 15-26.

4. Fuchs, G., et al., "High trapped fields in bulk $\mathrm{YBa} 2 \mathrm{Cu} 3 \mathrm{O} 7-\delta$ samples at temperatures around $50 \mathrm{~K} . "$ Applied Physics Letters, 1997. 70(1): p. 117-119.

5. $\quad$ Fuchs, G., et al., "Trapped magnetic fields larger than $14 \mathrm{~T}$ in bulk YBa2Cu3O7-x." Applied Physics Letters, 2000. 76(15): p. 2107 2109.

6. Gruss, S., et al., "Superconducting bulk magnets: Very high trapped fields and cracking." Applied Physics Letters, 2001. 79(19): p. 3131-3133.

7. Ainslie, M.D., et al., "Modelling and comparison of trapped fields in (RE) BCO bulk superconductors for activation using pulsed field magnetization." Superconductor Science and Technology, 2014. 27(6): p. 065008.

8. Kim, C.-J., et al., "Nonuniform distribution of second phase particles in melt-textured $\mathrm{Y}-\mathrm{Ba}-\mathrm{Cu}-\mathrm{O}$ oxide with metal oxide $(\mathrm{CeO} 2, \mathrm{SnO} 2$, and $\mathrm{ZrO} 2)$ addition." Journal of Materials Research, 1995. 10(7): p. 1605-1610.

9. Endo, A., et al. "Macrosegregation of Y2Ba1Cu1O5 particles in Y1Ba2Cu3O7 $-\delta$ crystals grown by an undercooling method." Journal of Materials Research, 1996. 11(4): p. 795-803.

10. Shiohara, Y. and A. Endo, "Crystal growth of bulk high-T c superconducting oxide materials." Materials Science and Engineering: R: Reports, 1997. 19(1): p. 1-86.

11. Chow, J., et al., "Processing, Y2BaCuO5 distribution and critical current density in large grain Pt-doped YBCO." Materials Science and Engineering: $B, 1998.53(1-2)$ : p. 79-85.

12. Diko, P., "Growth-related microstructure of melt-grown REBa2Cu3Oy bulk superconductors." Superconductor Science and Technology, 2000. 13(8): p. 1202.

13. Diko, P., et al., "Growth related $\mathrm{Y} 2 \mathrm{BaCuO} 5$ particle concentration micro-inhomogeneity in the growth sectors of TSMG YBa2Cu3O7/Y2BaCuO5 bulk superconductor." Physica C: Superconductivity, 2003. 384(1): p. 125-129.

14. Shangguan, D., S. Ahuja, and D. Stefanescu, "An analytical model for the interaction between an insoluble particle and an advancing solid/liquid interface." Metallurgical and Materials Transactions A, 1992. 23(2): p. 669-680.

15. Goyal, A., et al., "Mechanical properties of highly aligned YBa2Cu3O7- $\delta$ effect of $\mathrm{Y} 2 \mathrm{BaCuOx}$ particles." Physica $C$ : Superconductivity, 1991. 183(4-6): p. 221-233.

16. Fujimoto, H., M. Murakami, and N. Koshizuka, "Effect of $\mathrm{Y} 2 \mathrm{BaCuO5}$ on fracture toughness of YBCO prepared by a MPMG process." Physica C: Superconductivity, 1992. 203(1): p. 103-110.

17. Mathieu, J.-P., et al., "The contribution of 211 particles to the mechanical reinforcement mechanism of 123 superconducting single domains." Superconductor Science and Technology, 2003. 17(1): p. 169

18. Matsui, M., et al., "Mechanical properties of $\mathrm{Nd}-\mathrm{Ba}-\mathrm{Cu}-\mathrm{O}$ bulk superconductors." Superconductor Science and Technology, 2003. 16(9): p. 1086.

19. Nariki, S., et al., "Effects of Dy2BaCuO5 contents on microstructure and mechanical strength of $\mathrm{Ag}$-added $\mathrm{Dy}-\mathrm{Ba}-\mathrm{Cu}-$ O bulk superconductors." Physica C: Superconductivity, 2004. 412: p. 651-656.

20. Murakami, A., et al., "Evaluations of mechanical properties in Dy123 single-grain bulk superconductors with low porosity." IEEE
Transactions on Applied Superconductivity, 2009. 19(3): p. 29952998.

21. Murakami, A., et al., "Distribution of mechanical properties in a single-grain Sm123 bulk superconductor at liquid nitrogen temperature." IEEE Transactions on Applied Superconductivity, 2006. 16(2): p. 1003-1006.

22. Murakami, A., et al., "Mechanical properties of $\mathrm{Sm}-\mathrm{Ba}-\mathrm{Cu}-\mathrm{O}$ bulk superconductors at room temperature." Physica C: Superconductivity, 2003. 392: p. 557-561.

23. Cardwell, D.A., "Processing and properties of large grain (RE) BCO.” Materials Science and Engineering: B, 1998. 53(1-2): p. 110.

24. Chen, Y., et al., "A new method for net-shape forming of large, single-domain YBa2Cu3O6+x." Physica C: Superconductivity, 1994. 234(3-4): p. 232-236.

25. Hondros, G., "The evaluation of Poisson's ratio and the modulus of materials of a low tensile resistance by the Brazilian (indirect tensile) test with particular reference to concrete." Australian Journal of Applied Science, 1959. 10(3): p. 243-268.

26. Namburi, D.K., et al., "An improved top seeded infiltration growth method for the fabrication of $\mathrm{Y}-\mathrm{Ba}-\mathrm{Cu}-\mathrm{O}$ bulk superconductors." Journal of the European Ceramic Society, 2016. 36(3): p. 615-624.

27. Jorgensen, J., et al., "Structural properties of oxygen-deficient

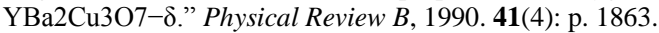

28. Abràmoff, M.D., P.J. Magalhães, and S.J. Ram, "Image processing with ImageJ." Biophotonics International, 2004. 11(7): p. 36-42.

29. Fairhurst, C. "On the validity of the 'Brazilian'test for brittle materials." International Journal of Rock Mechanics and Mining Sciences \& Geomechanics. 1964. Elsevier.

30. Weibull, W., "Wide applicability." Journal of Applied Mechanics, 1951. 103: p. 293-297.

31. Goyal, A., et al., "Anisotropic hardness and fracture toughness of

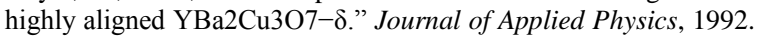
71(5): p. 2363-2367.

32. Lee, D. and K. Salama, "Enhancements in current density and mechanical properties of $\mathrm{Y}-\mathrm{Ba}-\mathrm{Cu}-\mathrm{O} / \mathrm{Ag}$ composites." Japanese Journal of Applied Physics, 1990. 29(11A): p. L2017.

33. Ikuta, H., et al., "Synthesis and superconducting properties of caxis aligned, single-domain $\mathrm{NdBCO} / \mathrm{Ag}$, melt-processed superconductors." Superconductor Science and Technology, 2000. 13(11): p. 1559.

34. Nanjangud, S.C., R. Brezny, and D.J. Green, "Strength and Young's modulus behavior of a partially sintered porous alumina." Journal of the American Ceramic Society, 1995. 78(1): p. 266-268.

35. Tinschert, J., et al., "Structural reliability of alumina-, feldspar-, leucite-, mica-and zirconia-based ceramics." Journal of Dentistry, 2000. 28(7): p. 529-535. 